\title{
Realizing broadband electromagnetic transparency with a graded-permittivity sphere
}

\author{
Lei Sun, Jie Gao, ${ }^{*}$ and Xiaodong Yang $^{\dagger}$ \\ Department of Mechanical and Aerospace Engineering, \\ Missouri University of Science and Technology, \\ Rolla, Missouri 65409, USA
}

\begin{abstract}
Broadband electromagnetic transparency phenomenon is realized with a well-designed gradedpermittivity sphere, which has an extremely low scattering cross section over a wide frequency range, based on the generalized Mie scattering theory and numerical simulation in full-wave condition. The dynamic polarization cancellation is revealed by studying the variation of the polarization with respect to the frequency. Furthermore, a properly-designed multi-shell sphere is also proposed and examined in order to reduce the rigorous conditions for realizing the broadband transparency in experiments.
\end{abstract}

\footnotetext{
* Electronic address: gaojie@mst.edu

† Electronic address: yangxia@mst.edu
} 


\section{INTRODUCTION}

Metamaterials, the artificial media with unit cells smaller than the wavelength, possess unique electromagnetic properties unavailable in nature. Besides the well-known property of negative refraction $[1,2]$ that leads to imaging beyond the diffraction limit $[3,4]$, electromagnetic transparency is one intriguing capability of metamaterials, which has been extensively studied in physics and engineering communities since it has significant impact in various fields, including optics, medicine, biology, and nanotechnology. In order to achieve the electromagnetic transparency phenomenon, several different mechanisms have been proposed and examined in the past years. Transformation optics [5, 6] suggests covering the object with a sophisticated design of heterogeneous and anisotropic metamaterial shell that can bend the incident electromagnetic wave around the cloaked object. Although the transformation optics has been well developed in theory [7-9] and demonstrated in experiments $[10,11]$, it is still difficult to implement due to the approximated ideal parameters, high material losses, and the single operating frequency [12]. On the other hand, the scattering cancellation [13-16], also known as the plasmonic cloaking, suggests a properly-designed material shell to reduce the scattering of the covered object near the plasmon resonance frequency in order to make the object-shell system transparent. The fundamental principle behind this mechanism is that the polarization of the shell is out of phase with respect to the polarization of the covered object, thus the total polarization of the object-shell system is close to zero to the probing electromagnetic wave. Although the scattering cancellation

for transparency is robust $[17,18]$ and can be achieved in experiments $[19,20]$, the operating frequency is still limited to the plasmon resonance frequency of the system. Furthermore, the electromagnetic transparency can also be accomplished by applying the active cloaking [21-25], in which the covered object is surrounded by active sources that can tailor the scattering automatically with respect to the properties of the probing electromagnetic wave. However, the active cloaking needs the whole prior knowledge about the probing electromagnetic wave, such as frequency and phase, in order to determine the response of active sources. Meanwhile, the operating frequency range of the active cloaking approach is also strongly limited due to the dimensions of the active sources.

In previous work, the electromagnetic scattering from a graded-permittivity sphere is theoretically studied based on the generalized Mie scattering theory and the broadband 
electromagnetic transparency is revealed under the quasi-static condition [26]. To further explore the physical mechanism dominating such unique phenomenon, the broadband electromagnetic transparency is realized and studied in the rigorous full-wave condition based on the numerical simulations. A dynamic polarization cancellation associated to the frequency variation of the probing electromagnetic wave is achieved in a well-designed sphere with graded-permittivity along the radial direction. Since scattering cross section is a general physical quantity to describe the degree of the electromagnetic transparency [27], such graded-permittivity sphere is designed to have an extremely low scattering cross section over a wide frequency range in order to obtain the broadband electromagnetic transparency. The dynamic polarization cancellation with respect to the variation of the frequency is clearly revealed from the polarization analysis of the graded-permittivity sphere. Furthermore, a multi-shell sphere with homogeneous and isotropic permittivity in each shell corresponding to the graded-permittivity sphere is also proposed and examined in order to reduce the rigorous conditions for realizing the broadband transparency in experiments.

\section{THEORETICAL ANALYSIS}

Consider a dilute metal-dielectric composite, in which the permittivity of the metallic solute is represented by a simple lossless Drude model $\varepsilon_{m}=\varepsilon_{\infty}-\omega_{p}^{2} / \omega^{2}$ and the permittivity of the dielectric solvent is denoted as $\varepsilon_{d}$, the effective permittivity of the composite can be approximately determined according to the simple mixing rule as $\varepsilon_{\text {eff }}=f_{m} \varepsilon_{m}+\left(1-f_{m}\right) \varepsilon_{d}=$ $f_{m}\left(\varepsilon_{\infty}-\varepsilon_{d}\right)+\varepsilon_{d}-f_{m} \omega_{p}^{2} / \omega^{2}$, where $f_{m}$ is the filling ratio of the metallic solute. The expression of the effective permittivity implies that if the filling ratio of the metallic solute can continuously vary along a certain direction in the metal-dielectric composite, the mixture can possess a graded effective permittivity in order to achieve broadband electromagnetic properties. Based on this idea, the scattering of a plane electromagnetic wave from an isotropic nonmagnetic sphere with the radius $r_{0}$ and a graded electric permittivity varying along the radial direction is studied, as depicted in Fig. 1(a). The graded electric permittivity tensor is described as $\varepsilon(\omega, r)=\varepsilon_{0} \varepsilon_{g}(\omega, r) \boldsymbol{I}$, in which $\varepsilon_{0}$ is the electric permittivity of the isotropic background medium (vacuum) and the relative permittivity $\varepsilon_{g}(\omega, r)$ is characterized by a simple lossless graded Drude model

$$
\varepsilon_{g}(\omega, r)=1-\omega_{p}^{2} / \omega^{2}\left[C_{0}-C_{1}\left(r / r_{0}\right)^{2}\right]
$$


and $\boldsymbol{I}=\boldsymbol{e}_{r} \boldsymbol{e}_{r}+\boldsymbol{e}_{\theta} \boldsymbol{e}_{\theta}+\boldsymbol{e}_{\phi} \boldsymbol{e}_{\phi}$ is the unit tensor in the spherical coordinates. In addition, parameters $C_{0}$ and $C_{1}$ are constants (graded parameters) controlling the variation of the electric permittivity, which are optimized as $C_{0}=0.5$ and $C_{1}=0.9$ in order to realize the broadband electromagnetic transparency according to the previous theoretical analysis [26]. The variation of the corresponding graded electric permittivity is illustrated in Fig. 1(b). It is clear that over the wide frequency range from zero to the plasma frequency $\omega_{p}$ the graded-permittivity sphere possesses both negative and positive permittivity along the radial direction, which leads to polarizations with opposite directions in the graded-permittivity sphere with respect to the incident electromagnetic wave. The opposite-direction polarizations can cancel each other out and result in a small dipole moment and therefore a small scattering cross section over a wide frequency range, so that the broadband electromagnetic transparency can be realized. In addition, it is worth mentioning that the radius of the graded-permittivity sphere is set as $r_{0}=\lambda_{p}$ associated with the plasma frequency $\omega_{p}$. Furthermore, the cancellation mechanism of the opposite-direction polarizations is not related to any resonance phenomena in the system, which means it is not strongly sensitive to the variation of the material loss [15], thus the material loss is ignored in order to emphasize the polarization cancellation phenomena in the graded-permittivity sphere.

Theoretically, the scattering cross section can be calculated via the generalized Mie scattering theory by introducing two scale potential functions $u(r, \theta, \phi)$ and $v(r, \theta, \phi)$ and expressing the electromagnetic field as [26]

$$
\begin{aligned}
\boldsymbol{E} & =\frac{1}{\mathrm{i} \omega \boldsymbol{\varepsilon}(\omega, r)} \boldsymbol{\nabla} \times \boldsymbol{\nabla} \times(\boldsymbol{r} u)+\boldsymbol{\nabla} \times(\boldsymbol{r} v), \\
\boldsymbol{H} & =\frac{1}{\mathrm{i} \omega \boldsymbol{\mu}(\omega, r)} \boldsymbol{\nabla} \times \boldsymbol{\nabla} \times(\boldsymbol{r} v)+\boldsymbol{\nabla} \times(\boldsymbol{r} u) .
\end{aligned}
$$

Subsequently, regarding the incident electromagnetic plane wave $\boldsymbol{E}_{\text {in }}=\boldsymbol{e}_{x} e^{\mathrm{i} k r \cos \theta}, \boldsymbol{H}_{\text {in }}=$ $\left(\boldsymbol{e}_{y} / Z\right) e^{\mathrm{i} k r \cos \theta}$, where $k=\omega \sqrt{\varepsilon_{0} \mu_{0}}$ and $Z=\sqrt{\mu_{0} / \varepsilon_{0}}$, the scale potential functions for the scattering field read [26]

$$
\begin{aligned}
& u_{\text {sca }}(r, \theta, \phi)=-\frac{\omega \varepsilon_{0}}{k} \sum_{\ell=0}^{\infty} \mathrm{i}^{\ell+1} \frac{2 \ell+1}{\ell(\ell+1)} A_{u}^{(\ell)} h_{\ell}^{(1)}(k r) P_{\ell}^{(1)}(\cos \theta) \cos \phi, \\
& v_{\text {sca }}(r, \theta, \phi)=-\frac{\omega \mu_{0}}{k Z} \sum_{\ell=0}^{\infty} \mathrm{i}^{\ell+1} \frac{2 \ell+1}{\ell(\ell+1)} A_{v}^{(\ell)} h_{\ell}^{(1)}(k r) P_{\ell}^{(1)}(\cos \theta) \sin \phi,
\end{aligned}
$$

with respect to the first kind spherical Hankel function $h_{\ell}^{(1)}(k r)$ and the associated Legendre polynomials $P_{\ell}^{(1)}(\cos \theta)$. On the other hand, the scale potential functions for the 
electromagnetic field in the graded-permittivity sphere read [26]

$$
\begin{aligned}
& u_{\mathrm{sp}}(r, \theta, \phi)=-\frac{\omega \varepsilon_{0}}{k} \sum_{\ell=0}^{\infty} \mathrm{i}^{\ell+1} \frac{2 \ell+1}{\ell(\ell+1)} B_{u}^{(\ell)} f_{u}(r) P_{\ell}^{(1)}(\cos \theta) \cos \phi, \\
& v_{\mathrm{sp}}(r, \theta, \phi)=-\frac{\omega \mu_{0}}{k Z} \sum_{\ell=0}^{\infty} \mathrm{i}^{\ell+1} \frac{2 \ell+1}{\ell(\ell+1)} B_{v}^{(\ell)} f_{v}(r) P_{\ell}^{(1)}(\cos \theta) \sin \phi
\end{aligned}
$$

in terms of the function $f_{u}(r)$ and $f_{v}(r)$ that are related to the confluent Heun function and the confluent hypergeometric function, respectively. Here the coefficients $A_{u}^{(\ell)}$ and $A_{v}^{(\ell)}$ (the scattering coefficients), as well as $B_{u}^{(\ell)}$ and $B_{v}^{(\ell)}$ are all determined by the boundary conditions at the surface of the graded-permittivity sphere. In addition, the scattering coefficients are directly associated to the scattering cross section

$$
C_{\text {sca }}=\frac{2 \pi}{k^{2}} \sum_{\ell=1}^{\infty}(2 \ell+1)\left(\left|A_{u}^{(\ell)}\right|^{2}+\left|A_{v}^{(\ell)}\right|^{2}\right)
$$

as a summation of an infinite series.

\section{NUMERICAL ANALYSIS}

In theoretical analysis, it is impossible to take all terms into account in the summation of Eq. (8), thus a properly determined cut-off term of the summation is very critical. Therefore, numerical simulation is performed to calculate the scattering cross section of the gradedpermittivity sphere. The efficiency factor of the scattering cross section $\sigma_{\text {sca }}$ (scattering efficiency for short), which is defined as the ratio of the scattering cross section and the cross section of the scatter itself, is examined based on the finite-element method (FEM). Figure 2(a) presents the variation of the scattering efficiency for the graded-permittivity sphere. For comparison, the scattering efficiency for a normal metallic-like sphere $\left[C_{1}=0\right.$ in Eq. (1)] is also calculated and plotted. It is shown that the scattering efficiency for the graded-permittivity sphere is extremely small compared with that of the normal sphere over the wide frequency range due to the cancellation of the opposite-direction polarizations of the graded-permittivity sphere. Especially, the cancellation of the opposite-direction polarizations also reduces the localized surface plasmon resonance on the graded-permittivity sphere, which leads to an extremely small scattering efficiency at the resonance frequency for the graded-permittivity sphere. However, strong localized surface plasmon resonance can be observed in the normal sphere. In order to reveal the polarization cancellation mechanism, the dipole moment of the graded-permittivity sphere is studied, which is defined as the 
integration of the polarization $\boldsymbol{P}$ [determined by the electric field in the graded-permittivity sphere with respect to Eqs. (2), (6), and (7)] over the volume of the graded-permittivity sphere

$$
\boldsymbol{p}=\int_{\Omega} \boldsymbol{P} \mathrm{d} V \approx \frac{\varepsilon_{\mathrm{eff}}-\varepsilon_{0}}{\varepsilon_{\mathrm{eff}}+2 \varepsilon_{0}} 4 \pi \varepsilon_{0} r_{0}^{3} \boldsymbol{E}_{\mathrm{in}},
$$

associated to the effective permittivity $\varepsilon_{\text {eff }}$ of the graded-permittivity sphere in the quasistatic limitation. The calculated absolute value of the dipole moment is depicted in Fig. 2(b). Since the scattering cross section in Eq. (8) can be described as

$$
C_{\mathrm{sca}} \approx \frac{128 \pi^{5} r_{0}^{6}}{3 \lambda^{4}}\left|\frac{\varepsilon_{\mathrm{eff}}-\varepsilon_{0}}{\varepsilon_{\mathrm{eff}}+2 \varepsilon_{0}}\right|^{2},
$$

the dipole moment in Eq. (9) is proportional to the square root of the scattering cross section. It is shown that the variation of the dipole moment as a function of the frequency in Fig. 2(b) is coincident with the variation of the scattering efficiency of the scattering cross section in Fig. 2(a) for both the graded-permittivity sphere and the normal sphere.

Besides the scattering efficiency, the scattering diagram provides detailed information about the scattering of the electromagnetic wave in terms of the distribution of the scattering electromagnetic field. Figure 3 plots the numerically simulated scattering diagram with the amplitude of the scattering electric field in both $x$-z plane and $y$ - $z$ plane for both the gradedpermittivity sphere [Fig. 3(a)] and the normal sphere [Fig. 3(b)] at different frequencies. In general, with respect to the same incident electromagnetic wave $\boldsymbol{E}_{\text {in }}=1 \mathrm{~V} / \mathrm{m}$, the scattering electric field by the graded-permittivity sphere is about one order of magnitude lower than the normal sphere. In addition, the scattering diagram of the graded-permittivity sphere performs ultra-compact patterns due to the polarization cancellation that manifests the broadband transparency. Specifically, according to the variation of the permittivity of the graded-permittivity sphere [Fig. 1(b)], it is clear that at the low frequency $\omega / \omega_{p}=0.34$ the graded-permittivity sphere behaves as a metallic-like sphere due to large part of the graded-permittivity sphere possesses a negative permittivity close to the core. Therefore, the scattering diagram represents a regular Mie scattering pattern but much more compact than that of the normal sphere because the positive permittivity of the graded-permittivity sphere near the sphere surface still causes the polarization cancellation. As the increasing of the frequency the polarization cancellation becomes more efficient that directly leads to a highly compact scattering pattern at the frequency of $\omega / \omega_{p}=0.55$. Finally, at the high frequency $\omega / \omega_{p}=0.82$, the polarization cancellation still impacts on the scattering pattern, 
but the high frequency incident electromagnetic wave also excites high order scattering that begins to demonstrate the scattering, thus the compact scattering pattern is different from a regular Mie scattering pattern. In contrast, the scattering diagram of the normal sphere is quite different and it clearly illustrates the variation from the Rayleigh scattering pattern to the Mie scattering pattern with the increased frequency.

The polarization cancellation can be demonstrated directly by exploring the distribution of the polarization of the graded-permittivity sphere, as illustrated in Fig. 4. Here the polarization of the graded-permittivity sphere is studied at three different frequencies that are coincident with the scattering diagrams in Fig. 3. The theoretically calculated polarization distributions [Fig. 4(a)] agree with the numerical simulation results [Fig. 4(b)] quite well. Moreover, it is clear that the polarization near the surface of the graded-permittivity sphere and the polarization near the core possess opposite direction with the zero-permittivity position as their boundary [as shown in Fig. 1(b)]. According to the permittivity profile described in Eq. (1), the zero-permittivity position in the graded-permittivity sphere varies with respect to the frequency so that the polarizations near the surface and the core will always possess opposite directions over a wide frequency range. Hence, the polarization cancellation effect will be satisfied at all frequencies for realizing the broadband electromagnetic transparency.

Furthermore, the graded-permittivity sphere can be realized as a multi-shell sphere with properly designed permittivity in each shell regarding the practical applications. According to the graded-permittivity profile shown in Fig. 1(b), a 10-shell sphere is constructed with each shell possessing the homogeneous and isotropic permittivity based on the mean value theorem as

$$
\varepsilon_{n}(\omega)=\frac{1}{\Delta r} \int_{r_{n-1}}^{r_{n}} \varepsilon_{g}(\omega, r) \mathrm{d} r=1-\frac{\omega_{p}^{2}}{\omega^{2}}\left(C_{0}-C_{1} \frac{r_{n+1}^{2}+r_{n+1} r_{n}+r_{n}^{2}}{3 r_{0}^{2}}\right),
$$

where $r_{n}=(n-1) r_{0} / 10$ for $n=1,2, \ldots, 10$ and $\Delta r=r_{n}-r_{n-1}$. The scattering efficiency of the 10-shell sphere is examined and displayed in Fig. 2(a), which is similar to that of the original graded-permittivity sphere except for small perturbations that may be caused by the discontinuous permittivity between the adjacent shells. Additionally, the polarization of the 10-shell sphere is also studied in Fig. 4(c) based on the numerical simulations. Similar to the graded-permittivity sphere shown in Fig. 4(b), the polarization of the 10-shell sphere also possesses opposite directions with respect to different frequencies and the polarization 
cancellation leads to the broadband electromagnetic transparency.

\section{CONCLUSIONS}

The broadband electromagnetic transparency is realized with a properly designed gradedpermittivity sphere with extremely low scattering cross section over a broad frequency range, using both the generalized Mie scattering theory and the numerical simulation in full-wave condition. The dynamic polarization cancellation is achieved with the graded-permittivity sphere in a wide frequency range for the demonstration of broadband electromagnetic transparency. A multi-shell sphere with properly arranged homogeneous permittivity in each shell is also proposed and studied to realize the broadband transparency phenomenon. This work can be a fundamental study to achieve the broadband object cloaking, and also will benefit many research areas in nanoscale optics and photonics such as optical sensing and photodetection.

\section{ACKNOWLEDGEMENT}

This work is partially supported by the Energy Research and Development Center at Missouri S\&T, the University of Missouri Research Board and Interdisciplinary Intercampus Research Program.

[1] Veselago V G 1968 The electrodynamics of substances with simultaneously negative values of $\varepsilon$ and $\mu$ Sov. Phys. Usp. 10, 509-14

[2] Shelby R A, Smith D R and Shultz S 2001 Experimental verification of a negative index of refraction Science 292, 77-9

[3] Pendry J B 2000 Negative refraction makes a perfect lens Phys. Rev. Lett. 85, 3966-9

[4] Liu Z, Lee H, Xiong Y, Sun C and Zhang X 2007 Far-field optical hyperlens magnifying sub-diffraction-limited objects Science 315, 1686

[5] Leonhardt U 2006 Optical conformal mapping Science 312, 1777-80

[6] Pendry J B, Schurig D and Smith D R 2006 Controlling electromagnetic fields Science 312, $1780-2$ 
[7] Leonhardt U and Philbin T G 2009 Transformation optics and the geometry of light Prog. Opt. 53, 69-152

[8] Leonhardt U and Tyc T 2009 Broadband invisibility by non-Euclidean cloaking Science 323, $110-2$

[9] Tyc T, Chen H Y, Chen C T and Leonhardt U 2010 Non-Euclidean cloaking for light waves IEEE J. Sel. Top. Quantum Electron. 16, 418-26

[10] Schurig D, Mock J J, Justice B J, Cummer S A, Pendry J B, Starr A F and Smith D R 2006 Metamaterial electromagnetic cloak at microwave frequencies Science 314, 977-80

[11] Valentine J, Li J, Zentgraf T, Bartal G and Zhang X 2009 An optical cloak made of dielectrics Nat. Mater. 8, 568-71

[12] Chen H S, Wu B I, Zhang B and Kong J A 2007 Electromagnetic wave interactions with a metamaterials cloak Phys. Rev. Lett. 99, 063903

[13] Kerker M 1975 Invisible bodies J. Opt. Soc. Am. 65, 376-9

[14] Chew H and Kerker M 1976 Abnormally low electromagnetic scattering cross sections J. Opt. Soc. Am. 66, 445-9

[15] Alù A and Engheta N 2005 Achieving transparency with plasmonic and metamaterial coatings Phys. Rev. E 72, 016623

[16] Alù A and Engheta N 2007 Cloaking and transparency for collections of particles with metamaterial and plasmonic covers Opt. Express 15, 7578-90

[17] Alù A and Engheta N 2007 Plasmonic materials in transparency and cloaking problems: mechanism, robustness, and physical insights Opt. Express 15, 3318-32

[18] Alù A and Engheta N 2008 Plasmonic and metamaterial cloaking: physical mechanisms and potentials J. Opt. A: Pure Appl. Opt. 10, 093002

[19] Edwards B, Alù A, Silveirinha M G and Engheta N 2009 Experimental verification of plasmonic cloaking at microwave frequencies with metamaterials Phys. Rev. Lett. 103, 153901

[20] Mühlig S, Cunningham A, Dintinger J, Farhat M, Hasan S B, Scharf T, Bürgi T, Lederer F and Rockstuhl C 2013 A self-assembled three-dimensional cloak in the visible Sci. Rep. 3, 2328

[21] Miller D A B 2006 On perfect cloaking Opt. Express 14, 12457-66

[22] Vasquez F G, Milton G W and Onofrei D 2009 Active exterior cloaking for the 2D Laplace and Helmholtz equations Phys. Rev. Lett. 103, 073901 
[23] Vasquez F G, Milton G W and Onofrei D 2009 Broadband exterior cloaking Opt. Express 17, $14800-5$

[24] Ma Q, Mei Z L, Zhu S K, Jin T Y and Cui T J 2013 Experiments on active cloaking and illusion for Laplace equation Phys. Rev. Lett. 111, 173901

[25] Selvanayagam M and Eleftheriades G V 2013 Experimental demonstration of active electromagnetic cloaking Phys. Rev. X 3, 041011

[26] Sun L and Yu K W 2011 Broadband electromagnetic transparency by graded metamaterials: scattering cancellation scheme J. Opt. Soc. Am. B 28, 994-1001

[27] Monticone F and Alù A 2013 Do cloaked objects really scatter less? Phys. Rev. X 3, 041005 


\section{FIGURE CAPTIONS}

FIG. 1. (Colour online) (a) Schematic diagram of the scattering of an incident plane electromagnetic wave by a graded-permittivity sphere located at the origin. The electromagnetic wave is propagating in the positive $z$-direction with the electric field in the $x$ direction and the magnetic field in the $y$-direction. (b) The variation of the permittivity of the well-designed graded-permittivity sphere with respect to the frequency, where the zero-permittivity position along the radial direction is plotted as the intersecting curve.

FIG. 2. (Colour online) (a) The variation of the efficiency factors of the scattering cross section of the graded-permittivity sphere (black), the normal sphere (red), and the 10-shell sphere (blue), with respect to the frequency. The circle markers indicate the numerical simulation results at different frequencies. (b) The variation of the absolute value of the dipole moments of the graded-permittivity sphere (black curve) and the normal sphere (reddashed curve), which is coincident with the variation of the efficiency factor shown in (a).

FIG. 3. (Colour online) The scattering diagram of (a) the graded-permittivity sphere and (b) the normal sphere at three different frequencies $\omega / \omega_{p}=0.34,0.55$, and 0.82 , with respect to the same incident plane electromagnetic wave of the electric field $\boldsymbol{E}_{\text {in }}=1 \mathrm{~V} / \mathrm{m}$. The scattering diagram is represented as the distribution of the electric field amplitude for the scattering electromagnetic wave in the $x-z$ plane (red) and the $y-z$ plane (blue), respectively. The electric field amplitude for the scattering electromagnetic wave from the graded-permittivity sphere is about one order of magnitude less than that from the normal sphere at all frequencies.

FIG. 4. (Colour online) The polarization of the graded-permittivity sphere with respect to the incident plane electromagnetic wave based on (a) the theoretical analysis and (b) the numerical simulation, as well as (c) the polarization of the 10-shell sphere based on the numerical simulation at the same three different frequencies in Fig. 3. The polarizations of the graded-permittivity sphere always possess opposite directions at all frequencies, leading to the polarization cancellation that results in the broadband electromagnetic transparency. 
(a)

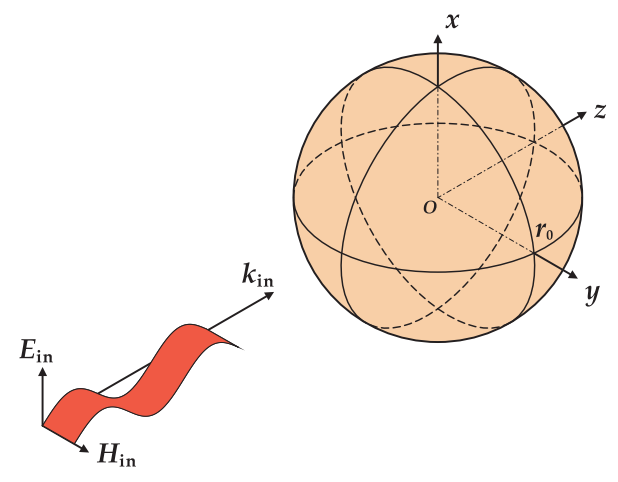

(b)

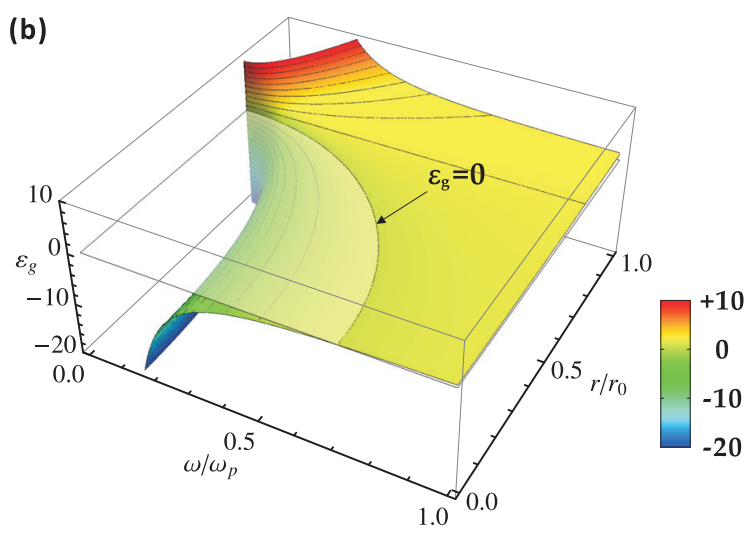

FIG. 1. Lei Sun, Jie Gao, and Xiaodong Yang 

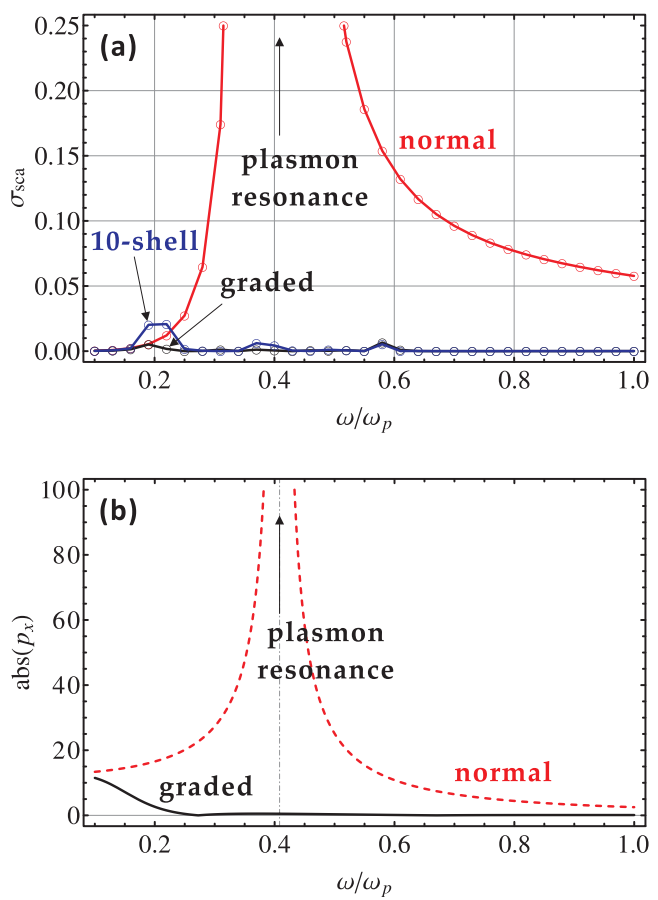

FIG. 2. Lei Sun, Jie Gao, and Xiaodong Yang 
(a)

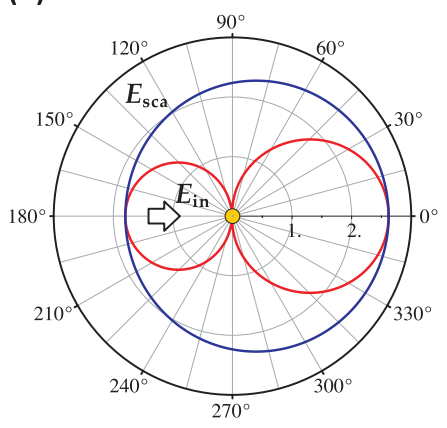

(b)

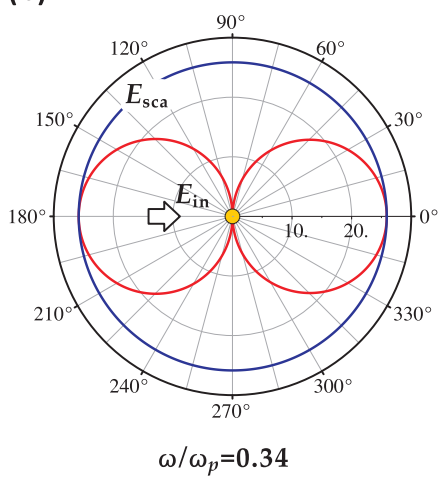

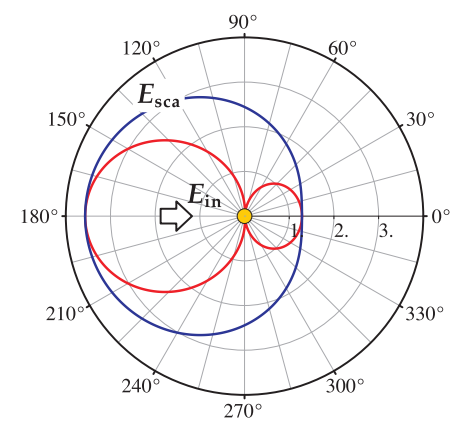

$E_{\text {in }}=1 \mathrm{~V} / \mathrm{m} \quad E_{\text {sca }}: \times 10^{-10} \mathrm{~V} / \mathrm{m}$
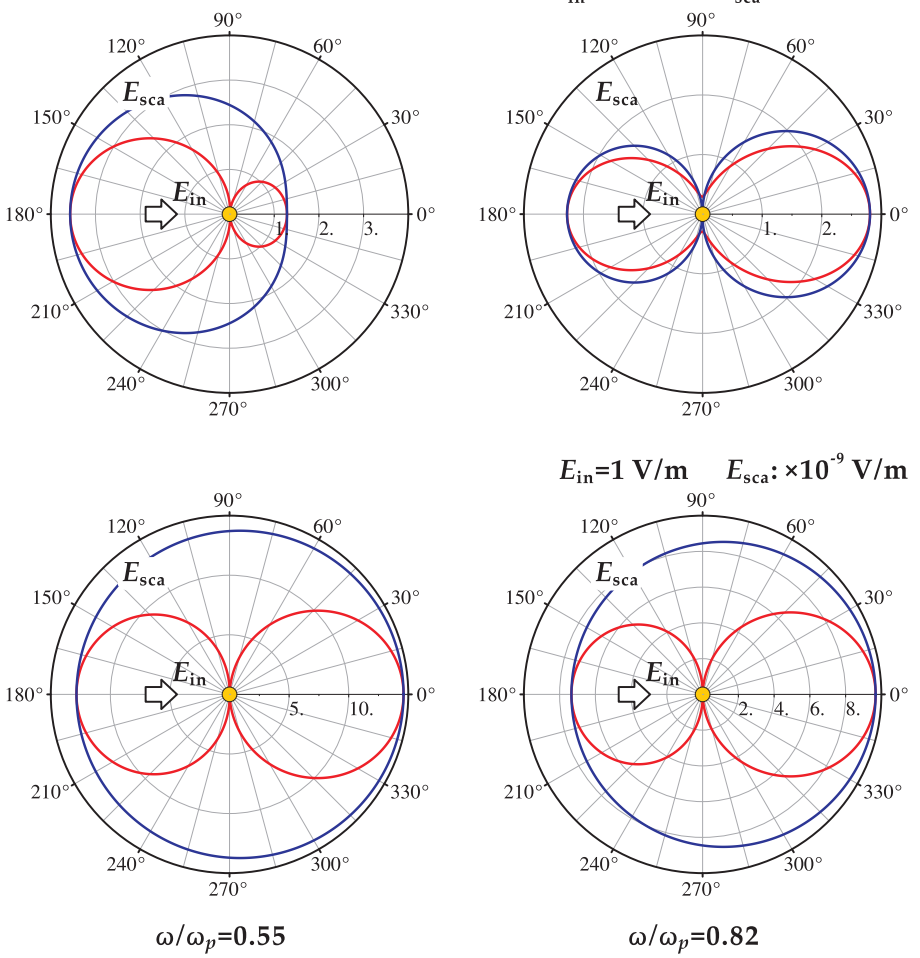

FIG. 3. Lei Sun, Jie Gao, and Xiaodong Yang 

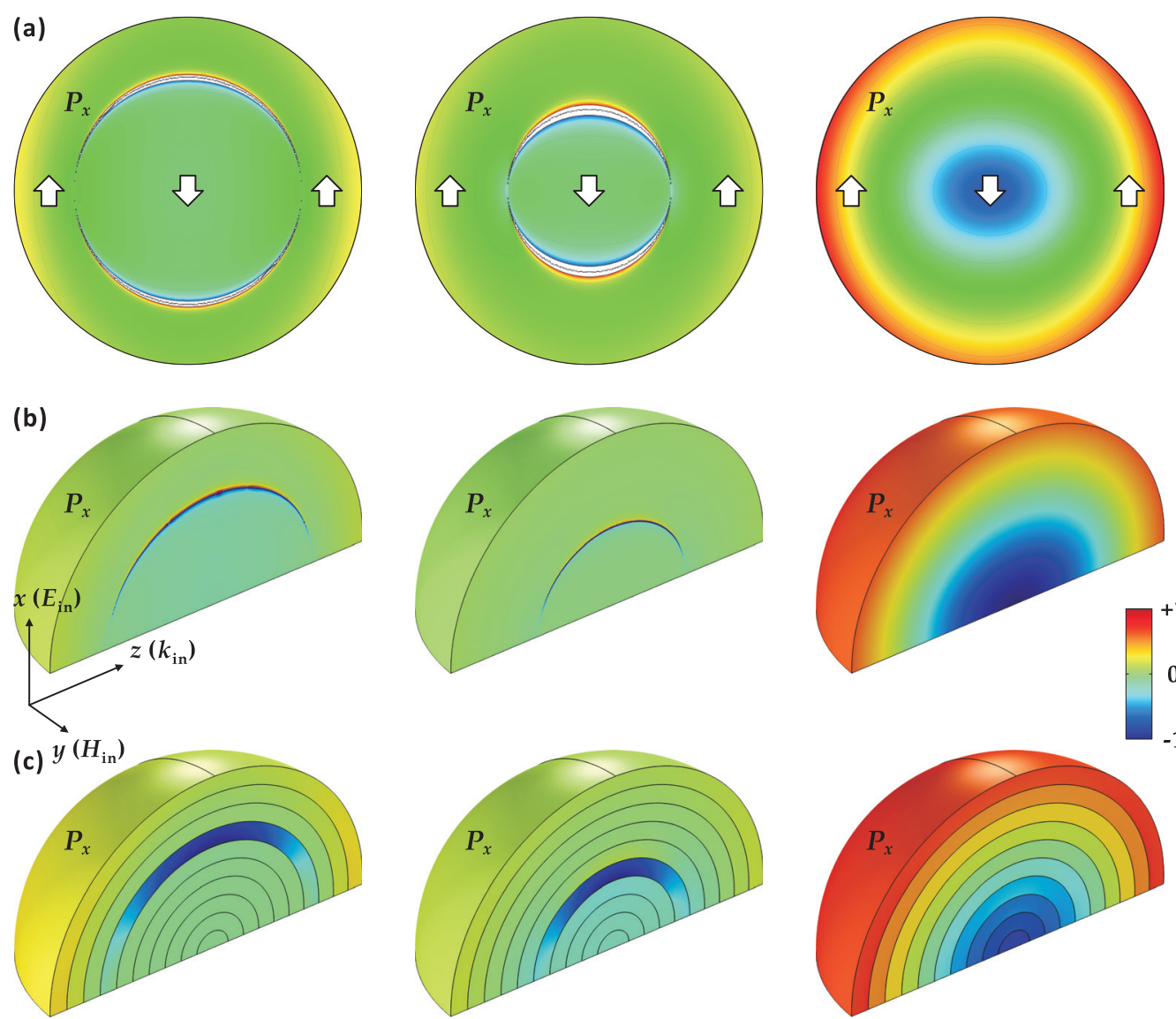

$\omega / \omega_{p}=0.34$

$\omega / \omega_{p}=0.55$

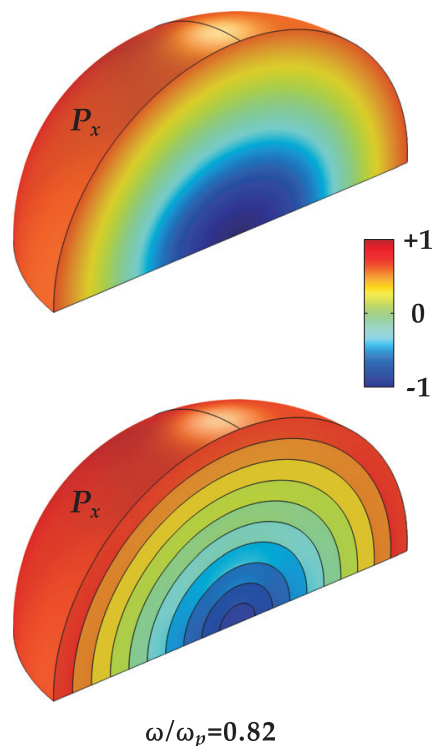

FIG. 4. Lei Sun, Jie Gao, and Xiaodong Yang 